\title{
Is There a Relationship Between Quadriceps Tendinopathy and Suprapatellar Plica? An Observational Case Series
}

\author{
Håkan Alfredson', Lorenzo Masci ${ }^{2,3}$, Christoph Spang $\mathbb{1 D}^{4,5}$ \\ 'Department of Community Medicine and Rehabilitation, Sports Medicine, Umeå University, Umeå, Sweden; ${ }^{2}$ Institute of Sports Exercise and Health, \\ University College Hospital London, London, UK; ${ }^{3}$ Sports \& Exercise Medicine, Queen Mary University of London, London, UK; ${ }^{4}$ Department of \\ Integrative Medical Biology, Anatomy Section, Umeå University, Umeå, Sweden; ${ }^{5}$ Private Orthopaedic Spine Center, Würzburg, Germany
}

Correspondence: Håkan Alfredson, Department of Community Medicine and Rehabilitation, Sports Medicine, Umeå University, Umeå, 90I87, Sweden, Tel +46 70228844I, Email hakan.alfredson@umu.se

Purpose: Chronic painful quadriceps tendinopathy is a relatively rare condition known to be difficult to manage. Conservative management is first-line treatment and if that fails open intra-tendinous revision surgery followed by a long rehabilitation period is used. There is sparse research on etiology and new treatment methods. This observational study aimed to evaluate the intra-articular findings in patients with chronic painful quadriceps tendinopathy resistant to conservative management.

Patients and Methods: Seven male athletes (mean age 33 years, range 22-40) suffering from chronic painful quadriceps tendinopathy in altogether 10 tendons, not responding to conservative management including heavy strength training, were included. Clinical examination and ultrasound scanning were used for diagnosis. Arthroscopy was used for evaluation of the inside of the knee. Results: In all 10 knees, there were obliterating major plica formations in the suprapatellar pouch.

Conclusion: Obliterating plica formations in the suprapatellar pouch may be involved in the aetiology and pathology in quadriceps tendinopathy.

Keywords: quadriceps, tendinopathy, suprapatellar, plica, surgery

\section{Introduction}

Chronic painful quadriceps tendinopathy is a rare clinical condition, most often seen in athletes involved in leg explosive sports. ${ }^{1-3}$ It is characterized by activity-related anterior knee pain with localized tenderness at the superior border of the patella. ${ }^{1,4}$ Overloading of the knee extensor mechanism is considered being a crucial etiological factor. ${ }^{1,5}$ Particularly repetitive squatting and prolonged knee flexion during activities such as weightlifting, football and volleyball have been associated with this condition. ${ }^{1}$ The diagnosis is based on clinical examination - pain on palpation of the quadriceps/ patella interface and resisted knee extension with the knee hyperflexed - and ultrasound or MRI detected changes (thickening and structural abnormalities) in the tendon. ${ }^{1}$

Little is known about how to assess and manage quadriceps tendinopathy. ${ }^{1}$ Non-operative treatment using different types of loading regimens is first-line treatment, and when surgery is needed intra-tendinous revision surgery is commonly used. ${ }^{1,4,6}$ Compared to the more common patellar tendinopathy, there is sparse scientific knowledge about the pain mechanisms related to this condition. Quadriceps tendinopathy is therefore often misdiagnosed as patellar tendinopathy. More research is needed to shed light on the pathogenesis and proper diagnosis of this very rare condition.

This observational study in patients with chronic painful quadriceps tendinopathy aimed to evaluate the intra-articular side of the quadriceps insertion and the surrounding tissues. 


\section{Materials and Methods}

\section{Patients}

Seven male patients (mean age 33 years [range 22-40]) with chronic painful quadriceps tendinopathy in altogether 10 tendons, were included. All patients had had a long duration ( $>6$ months) of pain symptoms from the quadriceps insertion and had tried conservative measures without success. All patients performed high frequencies of the following sport activities: weightlifting (2), soccer (1), alpine skiing (1), volleyball (1), cross-country skiing (1), swim-run (1).

\section{Clinical Examination and Ultrasound Imaging}

Clinical examination showed tenderness and loading pain in the quadriceps tendon insertion. US examination showed in all patients a thickening of the quadriceps tendon insertion, including tendinopathic changes with irregular fiber bundle structure and hypoechoic regions. There were also bony irregularities (spurs) in the tendon insertion into the patella.

\section{Surgical Procedure}

All patients were evaluated and treated with an US-guided arthroscopic approach performed in local anesthesia. In all patients, there were major plica formations in the suprapatellar pouch, often totally obliterating the pouch (Figure 1A and B). The obliterating plica formations in the suprapatellar pouch were shaved away with a $4.5-\mathrm{mm}$ shaver (Figure 1C). The portals were sutured, and $20 \mathrm{~mL}$ Chirocaine was injected into the joint. After surgery, patients were instructed to perform a structured rehabilitation with gradually increased loading.

There was Ethical approval for studies on the chronic painful tendon from the Regional Ethical Board at Umeå University.

\section{Ethical Consideration}

The study was carried out according to the principles of Declaration of Helsinki.

\section{Results}

In all patients, large plica formations totally or partly obliterating the patellar pouch were observed (Figure 1).

\section{Discussion}

In this small observational study on patients with chronic painful quadriceps tendinopathy resistant to conservative management, we found large, often totally obliterating, plica formations in the suprapatellar pouch during arthroscopy.

Suprapatellar plica formations in patients suffering from quadriceps tendinopathy have, to the best of our knowledge, never been described before. Quadriceps tendinopathy is a condition known to be difficult to treat, and there is no "golden standard" treatment. Therefore, these new findings can be of clinical relevance. We routinely use ultrasound and Doppler examination to diagnose tendinopathy, but in our hands, we cannot diagnose plica formations in the
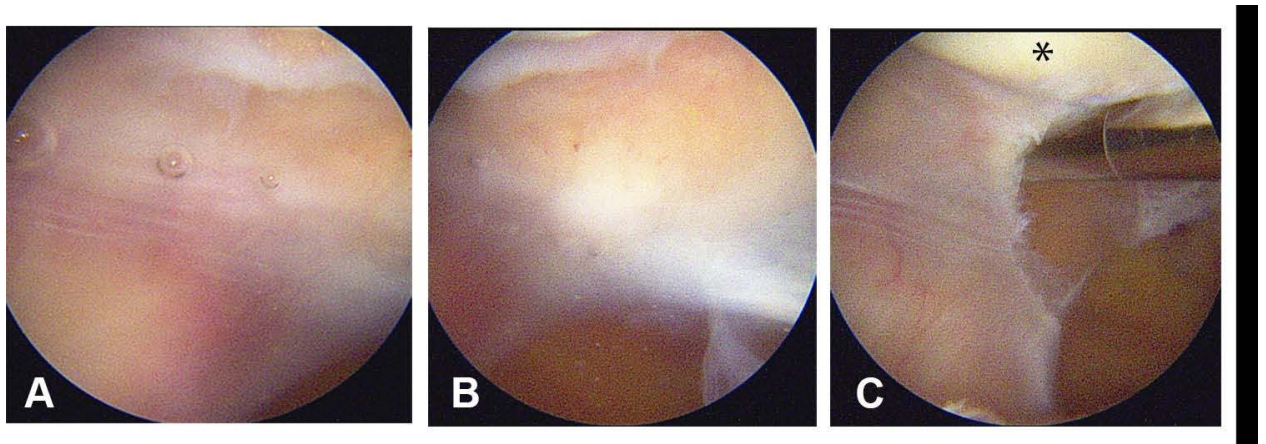

Figure I A plica formation obliterating the suprapatellar pouch in the knee in a patient with quadriceps tendinopathy. (A) Total obliteration. (B) Partial obliteration. (C) Shaver removing the whole plica. Asterisk indicates patellar. 
suprapatellar pouch. The reason to why we have started to perform arthroscopy for evaluation is that in our experience patellar cartilage defects is the most common co-existing diagnosis to quadriceps tendinopathy, and we have found that MRI does not reliably detect patellar cartilage defects. However, with the new findings from this observational study, maybe MRI should be used to diagnose the plica formations, but unfortunately, using MRI we still cannot be sure about cartilage defects in the patella.

According to literature quadriceps tendinopathy is a relatively rare condition, compared to the much more common patellar tendinopathy, and there are few scientific studies on this condition. ${ }^{1,4}$ It mostly affects athletes and rarely occurs in non-athlete individuals. ${ }^{4,7}$ The pain associated with quadriceps tendinopathy is generally believed to be associated with structural abnormalities inside the tendon, but there are, to the best of our knowledge, no studies verifying this. The findings of this case series are of interest since the pain mechanisms related to quadriceps tendinopathy are unknown, and treatment is known to be difficult. ${ }^{1,4,6}$ When conservative management fails, surgical treatment focuses on revision of the tendon inside. Via open surgery, and a longitudinal tenotomy, macroscopically abnormal tissue is excised. This treatment is often followed by a long (3-6 months) rehabilitation period. Unfortunately, there is no study showing longer term clinical results after this procedure.

Our experience from many years of treatment of tendinopathies is also that quadriceps tendinopathy is very rare compared to the much more common tendinopathies in the Achilles and patellar tendons. At our clinic, we see 1 quadriceps tendinopathy in 100 patellar tendinopathies. This condition responds poorly to eccentric training and sclerosing injection treatment. Because there are few cases compared to the other tendinopathies, the research on this condition has in our group been sparse. But with the good clinical results achieved using ultrasound-guided arthroscopic shaving on the deep side of the patellar tendon, we started to think about the deep side also for the quadriceps tendon.

During arthroscopic evaluation of a patient with quadriceps tendinopathy and suspected deep patellar cartilage defects, we found major obliterating plica formations in the suprapatellar pouch and normal patellar cartilage. After removal of the plica formations, opening up the suprapatellar pouch, the patient became pain-free and returned to full heavy knee-loading sport. This finding started interest in a possible relationship between obliterating plica formations in the suprapatellar pouch and quadriceps tendinopathy. After this single-case finding, we decided to use arthroscopy to evaluate patients with quadriceps tendinopathy. It surprised us to find the massive and obliterating plica formations in the suprapatellar pouch in all following patients. We used ultrasound examination to verify the diagnosis quadriceps tendinopathy before arthroscopy, and with that technology we cannot diagnose plica formations. MRI examination preoperatively would possibly have diagnosed the plica formations.

What is known about plica in the knee? There are two embryonal theories about plica formations in the knee. ${ }^{8}$ During embryonic development, synovial membranes divide the knee into 3 separate compartments (medial, lateral, and suprapatellar). Normally, these synovial membranes resorb and form a single cavity, but remnants of can form plicae. The other theory, early on the space between the distal femur and proximal tibial epiphysis is filled with mesenchymal tissue. Cavity opens up over time, but it is proposed that incomplete resorption of mesenchymal tissue cause failure of cavitation and leads to plica formation. The suprapatellar plica is the most common plica in the knee and originates from either the posterior quadriceps tendon or the anterior femoral metaphysis. ${ }^{8}$ In contrast to the more studied medial plica that is known to cause knee symptoms (eg, Medial Patellar Plica Syndrome) in 2-9\% of the cases, suprapatellar pouch is generally asymptomatic. ${ }^{9,10}$ Symptoms related to plica formations are known to be associated with inflammation secondary to trauma or repetitive stress on the tissue. ${ }^{8}$

From this knowledge about plica formations in the knee, it seems likely that they can be both part of the aetiology and the pathology. Obliteration of the suprapatellar pouch decreases the volume of the knee joint and likely changes the movement patterns of the patella, possibly affecting load tolerance in the quadriceps tendon. Overloading of the quadriceps tendon-repeated stress on the tendon tissue can cause inflammation and symptoms from the plica formations. For athletes active in sports with high demands on the knee extensors, this could likely cause problems, but for nonathletes this might not be problematic. Many theories can be discussed on the possible relationship between plica formations in the suprapatellar pouch and chronic painful quadriceps tendinopathy, and the findings in this study indicate a relationship. 
This observational study did not aim to study the results after plica formation removal, but the good clinical results, all patients were satisfied and returned to their sports, indicate that there might be a potential to cure the pain related to quadriceps tendinopathy by removing the plica formations. In a following study, we will prospectively study the clinical results after this procedure.

\section{Conclusion}

In patients with chronic painful quadriceps tendinopathy, large obliterating plica formations in the patellar pouch is a common finding. Further studies should evaluate if the plica formations are associated with the pain mechanisms in this condition.

\section{Disclosure}

The authors report no conflicts of interest in this work.

\section{References}

1. Simpson M, Smith TO. Quadriceps tendinopathy-A forgotten pathology for physiotherapists? A systematic review of the current evidence-base. Phys Ther Rev. 2011;16:455-461.

2. Lian OB, Engebretsen L, Bahr R. Prevalence of jumper's knee among elite athletes from different sports: a cross-Sectional study. Am J Sports Med. 2005;33:561-567. doi:10.1177/0363546504270454

3. Kuwabara A, Fredericson M. Narrative: review of anterior knee pain differential diagnosis (other than patellofemoral pain). Curr Rev Musculoskelet Med. 2021;14:232-238. doi:10.1007/s12178-021-09704-9

4. King D, Yakubek G, Chughtai M, et al. Quadriceps tendinopathy: a review-part 1: epidemiology and diagnosis. Ann Transl Med. $2019 ; 7(4): 71$. doi:10.21037/atm.2019.01.58

5. Pfirrmann CW, Jost B, Pirkl C, Aitzetmuller G, Lajtai G. Quadriceps tendinosis and patellar tendinosis in professional beach volleyball players: sonographic findings in correlation with clinical symptoms. Eur Rad. 2008;18:1703-1709. doi:10.1007/s00330-008-0926-9

6. King D, Yakubek G, Chughtai M, et al. Quadriceps tendinopathy: a review, part 2-classification, prognosis, and treatment. Ann Transl Med. 2019;7 (4):72. doi:10.21037/atm.2019.01.63

7. Zwerver J, Bredeweg SW, van den Akker-scheek I. Prevalence of Jumper's knee among nonelite athletes from different sports: a cross-sectional survey. Am J Sports Med. 2011;39(9):1984-1988. doi:10.1177/0363546511413370

8. Al-Hadithy N, Gikas P, Mahapatra AM, Dowd G. Review article: plica syndrome of the knee. J Orthop Surg. 2011;19:354. doi:10.1177/ 230949901101900319

9. Zmerly H, Moscato M, Akkawi I. Management of suprapatellar synovial plica, a common cause of anterior knee pain: a clinical review. Acta Biomed. 2019;90(12-S):33-38. doi:10.23750/abm.v90i11-S.8781

10. Zmerly H, Akkawi I, Citarella R, Ghoch ME. Clinical management of medial patellar plica syndrome: mini review from diagnosis to treatment. Curr Rheumatol Rev. 2020;16(1):9-11. doi:10.2174/1573397115666181123182633

International Medical Case Reports Journal

\section{Dovepress}

\section{Publish your work in this journal}

The International Medical Case Reports Journal is an international, peer-reviewed open-access journal publishing original case reports from all medical specialties. Previously unpublished medical posters are also accepted relating to any area of clinical or preclinical science. Submissions should not normally exceed 2,000 words or 4 published pages including figures, diagrams and references. The manuscript management system is completely online and includes a very quick and fair peer-review system, which is all easy to use. Visit http://www.dovepress.com/testimonials. php to read real quotes from published authors.

Submit your manuscript here: https://www.dovepress.com/international-medical-case-reports-journal-journal 